\title{
Motivating People in Smart Environments
}

\author{
Berardina De Carolis and Irene Mazzotta \\ ${ }^{1}$ Intelligent Interfaces Research Group \\ Dipartimento di Informatica, University of Bari \\ Via Orabona, 4 - 70125 Bari - Italy \\ \{decarolis,mazzotta\}@di.uniba.it
}

\begin{abstract}
In this paper we discuss the possibility to extend PORTIA, a persuasion system currently applied in human-agent dialogs, to support ambient persuasion. We have identified a fitness center as an appropriate smart environment in which ambient persuasion strategies can be applied. According to the Ubiquitous Computing vision, in the fitness center the user is surrounded by several connected devices that cooperate in the persuasion process, each of them with the most appropriate strategy, mode of persuasion, style of communication and ability of exploiting the kairos principle. To this aim we propose a multi-agent system able to support this distributed and intelligent approach to persuasion that allows to follow the user during the gradual change from the initial attitude to sustain of long term behaviours.
\end{abstract}

Keywords: Persuasion Systems, Ambient Intelligence, Multi-Agent systems.

\section{Introduction}

As stressed in Stock et al. [21] persuasion is a hot topic for intelligent interfaces since future interactive systems may have contextual goals to pursue which aim to induce and to convince the user to perform a specific action in the real world. It is feasible to imagine that persuasive technologies can be integrated into different aspects of daily life, and in this way they might have a greater persuasive power than traditional approaches to human-computer interaction. Under this perspective, the synergy between ambient intelligence and persuasion might be effective also because this solution, compared to traditional systems, could take the advantage to adapt the persuasion process, strategy and communication style to the context by using the kairos Principle [8].

In this paper, we present an approach to ambient persuasion [1] based on a combination of pervasive and distributed computation in which we aim at motivating people in the context of well-being. In particular we focus on how an intelligent environment may motivate the user to believe certain things, to behave in a certain way, or to abstain from performing certain actions, etc. This becomes important especially in certain kind of environments, such as those devoted to well-being, that intrinsically have this vocation. In fact, wellness is not limited to a single moment of people daily life - in which a person may consult a conversational agent or a web site in order to get advices or suggestions for improving life quality - but it is a continuous 
process along the temporal dimension and it is more central and peculiar in some environments than in others (i.e. fitness centers, food shops, homes, etc.). Moreover, the devices in these environments may cooperate in order to support people in achieving their goals.

Ambient Intelligence solutions may provide a great opportunity for achieving the aim of distributing and embedding persuasion and coaching strategies into the environments that the user attends, according to the Ubiquitous and Pervasive Computing vision [25], in order to apply persuasion methods and techniques usable through several devices and in different usage contexts.

Changing habits in the context of well-being is influenced by several -rational and emotional- factors depending on the context, that can be intended as: 'What the user is doing, Where is the user, With whom, When' [6]. Of course, attention should be paid to insure that arguments are relevant and strong to the user, especially in ambient intelligence context where it is essential to consider the conditions in which the message is communicated. Therefore, in our opinion, it is important not only to distribute the message through the existing devices in the environment and to adapt the persuasion strategy, the arguments and their expression to the user and the context, but it is also necessary that all the environments involved in the user's activities, task, etc, may communicate in order to cooperate to achieve the common goal of caring for the user.

To this aim, we propose a multi-agent architecture which includes different types of agents: (i) Sensor Agents -used in order to provide information about sensors parameters and context features (i.e. temperature, heart rate, humidity, presence of the user in a room, etc..); (ii) Device agents -typical of the environment- that manage the active devices in the environment (e.g. cardio fitness machines, public displays, mirrors, etc.) and convey to the user the training according to the context and the aim of the environment; (iii) D-Me agents [4], represent the users in the environment as a kind of digital image of the user; finally, (iv) the persuader agent that we call Coach agent, decides the most promising persuasion strategy to apply in a given context and communicate the action plan to Device agents.

In order to show how this architecture works, we will consider a fitness center as a suitable place to test the approach. In fact, a fitness center is equipped with enough technology for simulating a smart environment, the users are already confident with the technology during their workout and, moreover, most of them want to be constantly motivated in order to reach their goals concerning an healthier lifestyle [15].

The paper is structured as follows. In Section 2 we discuss the relation between ambient persuasion and wellbeing. Section 3 describes the proposed architecture of the system. Then, in Section 4, we illustrate a scenario example that is used to show the functioning of such a system. A final discussion and future work directions are reported in Section 5. 


\section{Ambient Persuasion and Wellbeing}

Persuasion is a form of social influence and a ubiquitous part of contemporary life. It is a relatively new trend in the research community that shows a growing interest also into intelligent information technologies, and for better or for worse, persuasive technologies are already part of the everyday technological landscape (see examples in [8]). Coming from persuasion and technology, persuasive technologies are not exempt from ethical issue: they should be employed to change people's attitudes or behaviour without coercion or deception, acting therefore upon users' beliefs always in an atmosphere of free choice, where they are autonomous and able to change their mind. An application area in which persuasion can be used with great effectiveness is well-being, especially when its purpose is to persuade people to adopt a healthier diet, lifestyle, etc. In our opinion, wellness is a domain in which ambient persuasion technologies may increase its potential of alleviating the users' problem by helping them in triggering the decision to change their wrong habits and motivating them to achieving their goals. There are different examples of systems aimed to persuade in this application domain. Many of them are implemented as Embodied Conversational Agents that play a role aiming at inducing behavioural change in users, a role that traditionally was filled by coaches or therapists $[2,5,12]$.

Currently, the most common persuasive systems used in fitness centers (at least in Italy) employ the feedback mechanism to show to users effects of the exercise (i.e. Polar Cardio or Cardio Fitness machines and so on) and are generally isolated without the ability to communicate and cooperate with other devices in order to achieve the common goal of taking care of the user. In addition, a fitness center has professionals responsible for this purpose, as personal trainers and wellness coaches: they have a very important role in helping the user to change their habits and find the motivations to work hard for achieving their goals. However, beside that they can be expensive or unavailable when users need them, many people feel shame and fear of being judged by their human coach: sometimes this can be a motivation for changing attitude, sometimes it may compromise the success of the coaching strategy, increasing the user's attitude at overcoming barriers -especially emotional- and decreasing selfesteem. Several coaching systems have been implemented on mobile devices (see for example, My Weight Loss Coach for Nintendo DS, Nokia Fitness Coach for Nokia phones and the so many sport trackers like Endomondo for the most popular mobile platforms, or CardioTrainer for Android, or Sports-Tracker for Nokia) aimed at monitoring, supporting and tracking users' progress and improving their energy balance. Again, in many of them the user has to input data about her workout, eating behaviour, etc. On the contrary, in other systems, as Nintendo Wii fit, My Body Coach by BigBen Interactive, or Your Shape: Fitness Evolved, the new edition of Ubisoft's training software for Microsoft console, the user is monitored and motivated during the exercise even though these are not integrated with other daily activities and situations of the user.

According to [10], when persuasion is used in ambient intelligence contexts it may take advantage of the distributed intelligence of the environment in order to improve the effectiveness of the persuasion process. For instance, since entities taking part of the persuasion process are multiple the system may use repetition for increasing 
compliance. Moreover, these multiple sources may have different roles in the process of persuading, motivating, sustaining the user and, therefore, may use different strategies.

Again, an intelligent environment is a social place and therefore, people may share personal experiences with others that have the same problems, goals, needs [19]. In this sense, perceived similarity through shared experience may have an effect on compliance [7].

Finally, the system should be perceived not has having a pure functional intelligence but has being an emotionally and socially intelligent actor that may monitor the user and intervene appropriately at the right moment.

In the light of these premises, we present an agent-based system that tries to apply the principles of ambient persuasion in a smart fitness center.

\section{The proposed System}

According to the ambient persuasion model proposed by Kaptein et al. [10], the first difference from traditional persuasive systems consists in the fact that the persuasion process can be distributed not only with respect of multiple sources but also according to the phases that constitute the gradual change from the initial attitude to sustain long term behaviour. In the application domain considered in this paper, the system provides a first phase in which the user should be persuaded to have the intention of adopting a certain behaviour, for instance a particular type of workout, and then, in a subsequent phase should be sustained using appropriate motivational cues, during the entire path of actuation of the suggested behaviour.

In order to generate the most appropriate persuasive message to the user, we extended PORTIA and used its reasoning and argumentation model. As far as the sustain phase it is necessary to reason on which motivational arguments have to be adopt for continuing to motivate the user according to the situation. To this aim, we started an empirical study aiming at exploiting the knowledge and rules that human personal trainer and fitness professionals use.

Before illustrating the architecture and the functioning of the system, let us introduce a brief overview of PORTIA.

\subsection{An overview of PORTIA}

PORTIA is a user-adapted persuasion system capable of simulating the persuasion process used by humans to convince someone to perform a given action. In this paper we provide a brief overview of the system. For a more detailed description of PORTIA, please refer to [14] It mainly focuses on two typical aspects of the human persuasion in order to produce effective persuasion attempt in different contexts: on one hand, the ability of reasoning on the potential strength of alternative persuasive strategies for a given user, in order to select the most appropriate one; on the other hand, the capability of combining rational and emotional modes of persuasion, 
according to the theory of a-rational persuasion [16]. The strategies represented in the model are the result of a combination of theoretical [22, 23, 18]) and empirical [13] background. The key points of the system are the separation between reasoning and argumentation phases in the persuasion process [24], and the use of Belief Networks to represent the uncertainty inherent in this form of practical reasoning [17].

PORTIA considers three knowledge bases: the User Model, the Persuasion and the Argumentation Knowledge Bases.

The User Model. Understanding the presumed weight of user's goals is crucial to select the most promising persuasion strategy in a given context. User Model is employed to reason about the user's presumed characteristics. Rather than acquiring this knowledge through direct questions, PORTIA attempts to implicitly infer it, with some level of uncertainty, from information about user's personality traits and living habits. The User Model includes a specific knowledge and a general knowledge component. The former collects facts about the user (evidence). The second represents criteria to infer the user's goals and abilities under conditions of uncertainty in the form of Elementary Belief Networks (EBNs) that are belief networks with only one leaf node representing uncertain implications. In particular, user's rational and emotional goals can be inferred respectively from knowledge about user's habits and personality traits.

The Persuasion Knowledge Base is employed to model rational and emotional strategies. The Persuasion model is defined in term of goals and beliefs from the Persuader's perspective that may employ rational as well as emotional strategies (but also a mixture of them) to induce the user to perform a given action. Persuasion strategies are represented with EBNs too. In particular, emotions may be introduced in the persuasion process in two forms: by selecting an emotional goal or by activating, through arousal of user's emotion, an intermediate goal which is instrumental to the final one. The PORTIA's persuasion strategies are summarized in Table 1. For more details see [15].

Table 1.A summary of the Persuasion Strategies used by PORTIA

\begin{tabular}{|c|c|}
\hline \multicolumn{2}{|c|}{ PORTIA's Persuasion KB } \\
\hline \multicolumn{2}{|c|}{ General induction of intentions } \\
\hline \multicolumn{2}{|c|}{$\left[\left(\right.\right.$ VGoal $\left.\cup g_{i}\right) \wedge\left(A G o a l \cup g_{i}\right) \wedge($ Bel $\cup$ Implies $(a, g i)) \wedge($ Bel $\cup$ CanDo $\left.(U, a))\right] \rightarrow ?(\operatorname{Int} \cup$ Do $(U$ a a $)) \quad[i]$} \\
\hline \multicolumn{2}{|c|}{$\begin{array}{l}\text { It may be summarized as follow: "If User has the goal } g \text { (VGoal U g) and it is really relevant at this time (AGoal U } \\
\text { g) and he believes that doing the action a implies achieving } g \text { in a more or less near future (Bel U Implies(a,gi)), } \\
\text { and he believes that has the ability to do a (Bel U CanDo(U,a)), then probably user intends to do a (Int U Do(U a))" } \\
\text { (from Miceli et al, 2006). }\end{array}$} \\
\hline Rational induction of intention & Emotional induction of intention \\
\hline$g_{i} \epsilon\{$ Rational goal set $\}$ & $g_{i} \in\{$ Emotional goal set $\}$ \\
\hline $\begin{array}{l}\text { It focuses on rational goals like 'to be in good health', } \\
\text { 'to have a good appearance', and so on. }\end{array}$ & $\begin{array}{l}\text { It focuses on rational goals like 'to make friends', to be } \\
\text { in good mood', and so on. }\end{array}$ \\
\hline \multicolumn{2}{|c|}{ Activation of goal strategy } \\
\hline \multicolumn{2}{|c|}{$\begin{array}{l}\text { Activation through a belief or an emotion of an intermediate goal which is instrumental to the user's goal. It } \\
\text { considers two possible applications: Rational Activation strategy or Emotional one. }\end{array}$} \\
\hline \multicolumn{2}{|c|}{ Induction of beliefs } \\
\hline ans-end implication. It rep & the action-goal relation. \\
\hline
\end{tabular}




\begin{tabular}{|l|l|l|l|l|l|}
\hline $\begin{array}{l}\text { Appeal to } \\
\text { Expert } \\
\text { Opinion }\end{array}$ & $\begin{array}{l}\text { Appeal to } \\
\text { Popular } \\
\text { Opinion }\end{array}$ & $\begin{array}{l}\text { Appeal to } \\
\text { Position to } \\
\text { Know }\end{array}$ & $\begin{array}{l}\text { Appeal to Friendly } \\
\text { Personal Experience }\end{array}$ & $\begin{array}{l}\text { Appeal to } \\
\text { Examples }\end{array}$ & Others \\
\hline
\end{tabular}

The Argumentation Knowledge Base is employed to translate each strategy into an argument. Items to include in the argument correspond to the variables associated with nodes of EBNs, and the way these items are combined in the message (order in which to present them and relationships among the various parts) is represented into Elementary Argumentation Plans (EAPs) that are a coherent translation of EBNs. EAPs are built on two theoretical grounds: Walton's Argumentation Schemes [20] and Rhetorical Structure Theory (RST) [11]. In particular, EAPs represent the association between rhetorical relations (RRs) and argumentation scheme.

PORTIA considers two main modules: the Reasoning module (REASONER), and the Argumentation one (ARGUER).In the Reasoning module, PORTIA exploits the information about the user (User Model KB), computes the degree of importance of the various -rational and emotional-goals on which focus the persuasion strategy, and evaluates the persuasiveness of different combination of strategies (Persuasion Strategies KB) and selects the most promising one with respect to the goal of inducing in the user the intention to do a certain action. For this purpose, PORTIA builds a complex Belief Network (BN) by dynamically chaining forward several EBNs. The BN is a representation of the user's mental state that enables to apply a "what-if" reasoning form for evaluating the persuasive power of the strategies, and to select the most promising one.

In the Argumentation module, PORTIA has to construct the arguments to express the strategy selected in the previous step. To this aim, PORTIA explores the complex Belief Network and decides the items to mention, their presentation order and the rhetorical relations among them. Also, she has to decide whether to include an appeal to cognitive consistency (between the user's of goals and beliefs, and his behaviour) as a form of encouragement to adopt a more consistent behaviour. That is, PORTIA has to translate the complex Belief Network into a coherent discourse plan. The discourse plan is dynamically built by combining the elementary argumentation plans (Argumentation Plans KB) that represent the elementary beliefs networks included in the Belief Network. The discourse plan is then translated into a natural language message used as an attempt to persuade the user.

\subsection{The System Architecture}

In order to develop a system for ambient persuasion in the context described in the Introduction, we propose an extension of a multi-agent platform implemented in another project [4] which considers four types of agents:

i) Sensor Agents - they are used in order to provide information about sensors parameters and context features (i.e. temperature, heart rate, humidity, presence of the user in a room, etc..).

ii) Device agents - they control the active devices in the environment (e.g. cardio fitness machines, public displays, mirrors, etc.) and communicate with the user 
by conveying the messages of the coach agent according to the display facilities typical of the controlled device.

iii) D-Me agents - they represent the users in the environment as a kind of digital alter-ego. In particular, a D-Me agent knows the user and monitors all his/her activities, when authorized, communicates the information required by the environment according to the privacy policies set by the user. Of course, the user can always decide which data to send to which environment and the level of detail of the information to be provided to the environment and the coach agent.

iv) Coach agents - they decide the most promising strategy to apply in a given context in order to persuade/motivate/sustain the user involved in the workout and communicate the action plan to the Device agents or to the D-Me agents. Coach agents are specialised in persuasion strategies typical of the environment.

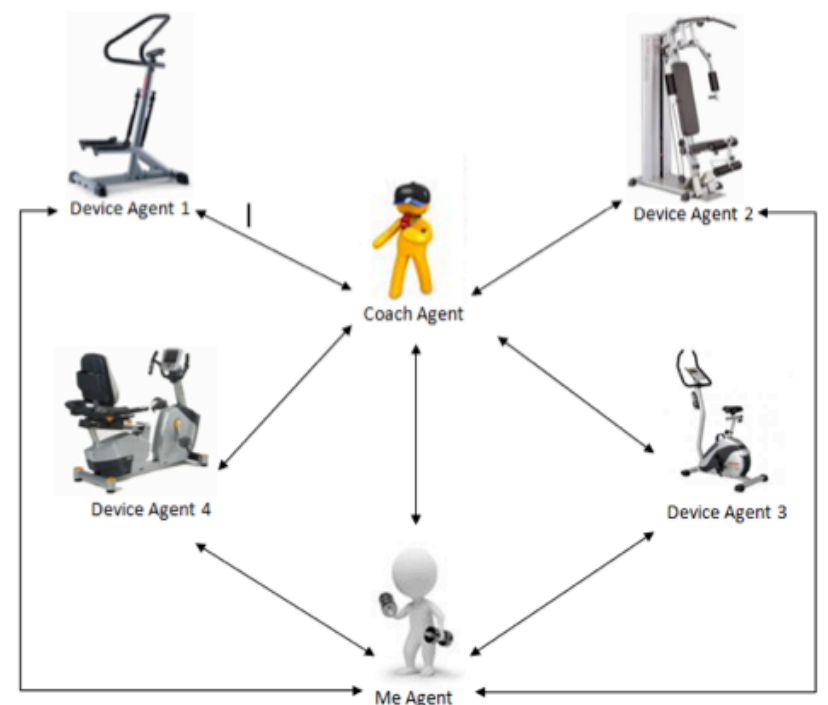

Fig. 1. A schema of a possible configuration of the multi agent platform.

It is worth noting that the architecture that we propose has not been conceived with the sole purpose of persuading the user, but it aims at implementing smart environments that aims at improving the quality of life of the user. In these environments all agents exchange data and information in order to provide services (recommendations, information, motivation) suitable for helping users in achieving their goals.

For instance, the D-Me agent, by monitoring the user behaviour, knows about his/her meals, and, through social networks, may know who are his/her friends, etc. Then, it may communicate this data to the Coach Agent that may adopt the optimal persuasion strategy and arguments accordingly. Again, suppose that the doctor recommended to the user to loose weight. When the user goes to the food shop with the intention to buy a sweet cream, the D-Me agent may communicate this 
information to the Coach Agent that may act to persuade him not to do it by adopting the most effective persuasion strategy and arguments.

For this reason, one might argue: why not delegating the entire ambient persuasion process to the D-Me agent? The idea is to build a platform environment-independent that may be applied in a fitness center as well as in a virtual home and, possibly, enabling interaction between different environments so as to support the user at different times of the day. According to this perspective, we believe that D-Me agent should not have specific knowledge of the environment or the technological devices because persuasion strategies and arguments used by a Personal-Coach in the fitness center are probably different from those applied by Personal-Butler in the smart home.

\section{An example}

The following example is a simulation of the system's behaviour in a typical scenario of the gym environment. Let us consider the following starting conditions.

Robert is a man below 40 years who regularly makes medical check-ups. He is a hypochondriac, too. He is probably an extravert because he feels comfortable around people. The doctor suggested him to make some physical activity regularly. He decides to go to the gym but he is quite sceptical about this.

When Robert enters in the gym his D-Me agent has the permission to communicate to the other agents in the environment the anagraphical and physiometric data and other information about the user social network. Robert registers himself to the gym information system. Then, the system provides a personalized workout schema to Robert and, in order to persuade him to adopt the proposed workout, the Coach agent generates and communicates the following persuasion message, according to the $\mathrm{BN}$ in Figure 2:

"Hi Robert, I am your personal coach and this is your personalized workout schema. You should do it because I know that you care for your health and training has a lot of benefits on your health. In fact, the World Health Organization says that this is very important for health and it is a specialized agency of the United Nations that acts as a coordinating authority on international public health, it is an authoritative voice. In addition working out may be a great opportunity to know new friends. In fact, this is well known to all who attend gyms and there is no evidence against it. Come on! I'm sure you can do it if you wish". 


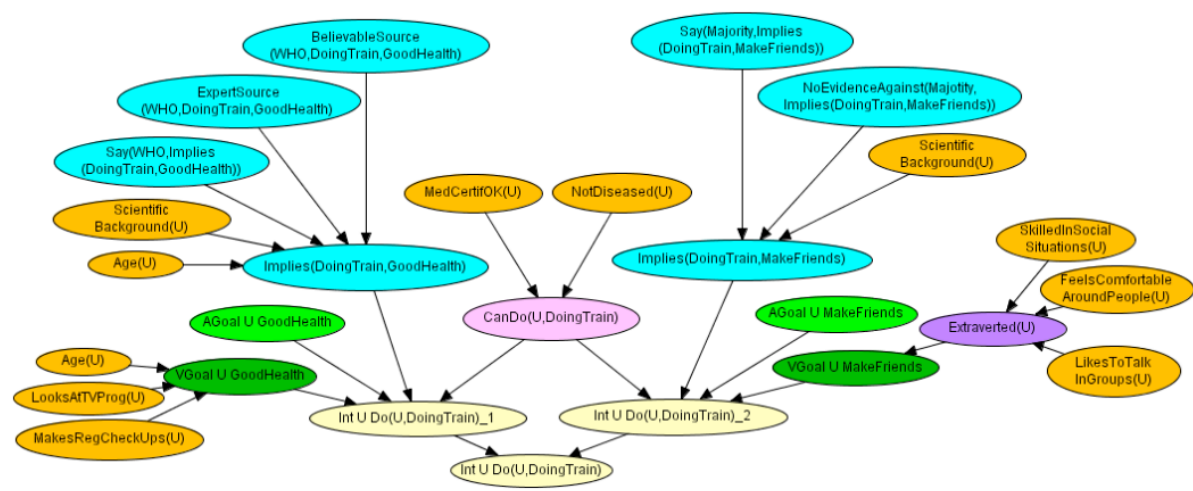

Fig. 2. The BN used by the Coach agent to simulate the effect of selected strategy on the user's mental state.

Figure 2 represents the result of the reasoning process of the persuasion model applied by the coach agent when tries to persuade the user to adopt the proposed workout. That is, the coach's representation of the user's mental state on which has been tested the effectiveness of the persuasion strategy. Reasoning component has propagated the user's evidence received by the D-Me agent into the EBN-KB and has inferred that, although 'to be in good health' is the presumed most important goal to Robert, the associated rational persuasion strategy does not seem to induce in the user the desired level of intention to do the proposed workout schema and a mixed strategy could be more effective. Therefore, Coach agent selects the goal with the highest value among the emotional goals and infers that the two candidate goals on which the persuasion strategy focuses are the rational goal to be in good health and the emotional goal to make friends. Moreover, it selects the belief induction strategies through appeal to expert opinion and appeal to popular opinion, respectively, for the goals of being in good health and making friends.

In a dialog perspective, persuasion, rather then a predefined, integrated set of propositions, is seen as a sequence of moves in which two parties (Persuader and Receiver) are reasoning together on some argument [9]. While monologic persuasion is characterized by the three steps (planning, plan revision and surface realization), in the 'pure' persuasion dialogues the sequence of exchanges includes some typical phases, and forms of reasoning, by the Persuader (that is, making a proposal, observing, classifying and reasoning on the Receiver's reaction and replying to it). Therefore, a persuasion attempt may be criticized by the Receiver in several ways: by questioning the goal premises; by attacking them with counter-arguments alleging that one or more of them is false; by undercutting the inferential link between premises and conclusion with critical questions; by rebutting the practical reasoning inference with counter-arguments asserting that the conclusion is false or by putting forward a proposal arguing for a different action, and contending that the arguments for this opposed proposal are stronger. Persuader must be able to respond appropriately to all these situations.

For example, let's suppose that Robert is hypochondriac and he does not want to do the proposed workout schema because he is afraid of getting sick. The Coach agent 
classifies this reaction as an object on the user's capability and, after reasoning on the $\mathrm{BN}$ in order to select the most appropriate response, tries to reassure him because all the tests say he's fine:

"I know you're scared but try to stay calm because your medical certificate says you're fine!"

Finally Robert goes to the bike and began his training.

Another strategy applied by the Coach agent is to motivate and sustain the user during the exercises. Let's suppose again that, despite the encouragement received, Robert starts going very slowly on the bike. Then the Device agent that monitor the user's exercises, communicates to the Coach agent the new situation of Robert. Then, the Coach tries again to reassure the user by conveying a following message on the display of the bike device:

"Robert, come on, do not worry, I'm tracking your heart and, at present, you are even below the threshold workout. Therefore, you can do more without fear of forcing your heart ... I monitor you, do not worry".

Robert starts to push a little more and retakes the right pace.

Then, finally, the user finishes the exercise. The Device agent transmit this information to the Coach that tells Robert that everything went well but that, the next time, he could do more.

\section{Discussion}

This contribution shows a preliminary work towards the development of a system for ambient persuasion in a fitness center based on a multiagent architecture. In the proposed architecture there are two agents that are central to the persuasion process. The D-Me agent, which manages the personal user profile, may transmit to the intelligent environment data about the user that may be important for adapting the persuasion strategy and the motivational messages. The Coach agent, which acts as a personal trainer, has the role of persuading the user to train and adopt a certain workout and also to sustain this behaviour during training. The Coach agent uses the reasoning and argumentation model of PORTIA for generating the persuasive message. While, for the generation of motivational messages to be provided in the sustain phase, we are collecting data from professionals expert in the fitness and wellness domain, such as personal trainers.

We are aware that in this domain the risk of producing a message that is not appropriate to the situation because of an inferential error on the user goal, personality traits, and so on may determine the selection of a wrong strategy or arguments and consequently may cause distrust in the user. In this case it is necessary to endow the environment with a formal model of trust [3], in order to give to the coach agent the capability of assessing the level of trust that the user has in the system behaviour and to reason on the cognitive factors involved on this project in order to recover the situation.

At moment, in order to test the effectiveness of the proposed system we used the knowledge of two personal trainers. To these people we proposed some scenarios, 
like the one in Section 4 of this paper, with the aim of collecting examples of motivational sentences, arguments to be used to motivate the users. Now we are conducting an experimental study that involves a greater number of experts in the fitness domain aiming at understanding:

- which are the features of the user relevant for adapting the motivational message;

- when to intervene with a motivational message;

- which are the strategies, at the reasoning and argumentation levels, most widely used according to the user features.

At present, the collected data give us some useful information for understanding how human personal trainers build in their mind the models of their clients and which are the features of the clients that influence their decision about how to motivate them.

For instance, the gender of the client seems to be important for choosing the arguments to use. Personality traits (mainly the levels of sociability and extraversion) influence the message style. While the cultural background, the age and the profession of the client influence the argumentation schema to be used to support some concepts and claims.

Moreover, from this initial analysis, seems clear that personal trainers initially classify their clients into stereotypes (Lazy, Super, Model, Normal, Sociable, ...) that help in deciding how to motivate them initially. This capability is related to the level of experience of the trainer.

Then, in our future work we plan to analyze the collected data and build the initial knowledge of the coach agent relative to stereotypes and reasoning rules in order to generate motivational messages appropriate to the user and to the situation. Moreover, we intend to give to our coach the capability to learn from the user feedback in order to refine the rules driving the choice of the optimal strategy.

\section{References}

1. Aarts, E., Markopoulos, P., de Ruyter, B.: The persuasiveness of ambient intelligence. In M. Petkovic, W. Jonker (Eds.), Security, privacy, and trust in modern data management. (pp. 367-381) Berlin, Germany: Springer (2007)

2. Bickmore, T:"Relational Agents: Effecting Change through Human-Computer Relationships" PhD Thesis, Media Arts \& Sciences, Massachusetts Institute of Technology (2003)

3. Castelfranchi, C., Falcone, R., Lorini, E.: A non-reductionist Approach to Trust. In J. Goldbeck (Ed.), Computing with Social Trust . Berlin, Springer, 45-72 (2008).

4. Cozzolongo, G., De Carolis, B., Pizzutilo, S.: A Personal Agent Supporting Ubiquitous Interaction. WOA 2004. Torino, Italia 2004: 55-61 (2004)

5. de Rosis, F., Novielli, N., Carofiglio, V., Cavalluzzi, A., De Carolis, B.: User Modeling And Adaptation In Health Promotion Dialogs With An Animated Character. International Journal of Biomedical Informatics, 514-531 (2006)

6. Dey, A. K., Abowd, G. D.: Towards a Better Understanding of Context and ContextAwareness. Workshop on 'The What, Who, Where, When, and How of ContextAwareness', as part of CHI 2000, The Hague, The Netherlands, (2000)

7. Festinger L (1954) A theory of social comparison processes. Hum Relat 7:117-140 
8. Fogg, B.J.: Persuasive Technology: Using Computers to Change What we Think and Do. Morgan Kaufmann (2002)

9. Guerini M., Stock O., Zancanaro M., O'Keefe D.J., Mazzotta I., de Rosis F., Poggi I., Lim M. Y. \& Aylett R. "Approaches to Verbal Persuasion in Intelligent User Interfaces". In P. Petta, R. Cowie and C. Pelachaud (eds.) The HUMAINE Handbook on Emotion-Oriented Systems Technologies. Springer, (2011).

10. Kaptein, M., Markopoulos, P., de Ruyter, B. \& Aarts, E. (2009). Persuasion in Ambient Intelligence, Journal of Ambient Intelligence and Humanized Computing.

11. Mann, W. C., Matthiesen, C. M. and Thompson S. A.: Rhetorical structure theory and text analysis. Information Sciences Institute Research Report ISI/RR-89-242, 89-242, (1989).

12. Marsella, S. C., Johnson, W. L., LaBore, C. M.: Interactive pedagogical drama for health interventions (2003)

13. Mazzotta, I. and de Rosis, F.: Artifices for persuading to improve eating habits. AAAI Spring Symposium on "Argumentation for consumers of health care". Stanford, USA. Technical Report SS-06-01, 76-85 (2006).

14. Mazzotta, I., de Rosis, F., Carofiglio, V.: PORTIA: a user-adapted persuasion system in the healthy eating domain. IEEE Intelligent Systems, 22, 6, 42-51 (2007)

15. Mazzotta, I., Silvestri, V., and de Rosis, F.: Emotional And Non Emotional Persuasion Strength. Proceedings of AISB'08, Symposium on 'Persuasive Technology', 14-21 (2008)

16. Miceli, M., de Rosis, F., Poggi, I.: Emotional and non-emotional persuasion. Applied Artificial Intelligence: an International Journal, 20, 10, 849-880 (2006)

17. Pearl, J.: Probabilistic Reasoning in Expert Systems: Networks of Plausible Reasoning. San Mateo, CA: Morgan Kaufmann (Pubs.) (1988).

18. Petty, R. E., and Cacioppo, J. T.: The elaboration likelihood model of persuasion. In L. Berkowitz (Ed.), Advances in experimental social psychology, 19, 123-205. New York: Academic Press (1986).

19. Pinel EC, Long AE, Landau MJ, Alexander K, Pyszczynski T (2006) Seeing I to I: a pathway to interpersonal connectedness. J Pers Social Psychol 90:243-257

20. Reeves B, Nass C (1996) The media equation: how people treat computers, television, and new media like real people and places. Cambridge University Press, Cambridge

21. Stock O, Guerini M, Zancanaro M.: Interface design and persuasive intelligent user interfaces. chapter The foundations of interaction design. Lawrence Erlbaum, Hillsdale, NJ, (2006)

22. Walton, D. N.: Argumentation Schemes for Presumptive Reasoning. Mahwah, N. J.,Erlbaum (1996).

23. Walton, D. N.: The place of emotion in argument. The Pennsylvania State University Press (1992).

24. Walton, D.: What is reasoning? What is an argument? Journal of Philosophy, 87, 399-419 (1990).

25. Weiser, M.: Some computer science issues in ubiquitouscomputing. Commun. ACM, 36(7):75-84 (1993) 